\title{
Combinatorial Characterization of Interference Coupling in Wireless Systems
}

\author{
Holger Boche, Fellow, IEEE, Siddharth Naik, and Martin Schubert, Member, IEEE
}

\begin{abstract}
We provide a combinatorial characterization of interference coupling in wireless systems, with the intent of obtaining a better insight into interference coordination and management. We introduce two bipartite graphs, namely the power graph and interference graph. We utilize these graphs and global dependency matrix containing only binary ( 0 and 1 ) entries to capture the effects of interference coupling in communication systems. We show that the irreducibility of the global dependency matrix $G$ is related to the connectivity of the power graph and the irreducibility of the matrix $G G^{T}$ is related to the connectivity of the interference graph. We prove that for strictly positive and strictly log-convex interference functions, the irreducibility of the matrices $G$ and $G G^{T}$ are necessary and sufficient conditions for the considered utility sets to be strictly convex. In this case there exists a unique optimizer for the problem of maximizing the product of utilities. We show that an interference balancing function is strictly log-convex, if and only if matrices $G$ and $G G^{T}$ are irreducible. We provide a simple yet comprehensive combinatorial characterization of interference coupled systems which abstracts away certain complexities of the physical layer.
\end{abstract}

Index Terms-Interference coupling, power graph, interference graph, global dependency matrix.

\section{INTRODUCTION}

$\mathbf{T}$ HE performance of the users in a multi-point to multipoint communication system is often influenced by interference coupling of the involved users. Investigating interference management and coordination could provide a means for delivering better performance to the users and in turn increasing the revenue of the operator. Furthermore, interference management and coordination for indoor wireless systems has not as yet been completely addressed.

We present a combinatorial characterization of interference coupling in wireless systems. A combinatorial characterization could be comprehensive and still a fairly simplistic framework to capture the effects of interference coupling in wireless networks. A combinatorial characterization has

Paper approved by N. C. Beaulieu, the Editor for Wireless Communication Theory of the IEEE Communications Society. Manuscript received February 4, 2009; revised July 28, 2010.

Holger Boche is with the Chair of Theoretical Information Technology, Technical University of Munich Arcisstrasse 21, 80331 Munich, Germany. He was previously with the Technical University of Berlin and the Heinrich Hertz Institute (e-mail: boche@tum.de).

Siddharth Naik is with the Chair of Theoretical Information Technology, Technical University of Munich Arcisstrasse 21, 80331 Munich, Germany. He was previously with the Technical University of Berlin and the Heinrich Hertz Institute (e-mail: sid.naik@tum.de). He was supported by the DFG Projects BO 1734/23-1 and BO 1734/24-1.

Martin Schubert is with the German Sino Lab for Mobile Communications, Heinrich Hertz Institute, Einsteinufer 37, 10587 Berlin (e-mail: schubert@hhi.fraunhofer.de). He was supported in part by the Bundesministerium fuer Bildung und Froschung $(B M B F)$ under grant 01SF0708 (project TEROPP).

Digital Object Identifier 10.1109/TCOMM.2011.050911.090072A a strong intuitive graph theoretic appeal and could provide another insight into existent power control algorithms, e.g. uniqueness of optimal solution outcome and convergence speed of the power control algorithms.

Consider a wireless system with $K \geq 2$ users from an index set $\mathcal{K}:=\{1, \ldots, K\}$. If the users are coupled by interference, then there is a general trade-off between the utilities $\boldsymbol{u}=\left[u_{1}, \ldots, u_{K}\right]^{T}$. We utilize an interference function framework [1] (defined in Section II-B) to capture the effects of interference coupling in wireless networks. The performance (utility) of each user is characterized by its signal-to-interference ratio (SIR), which is an important performance indicator in wireless systems [2]. The set of all feasible SIR levels with all users being active concurrently is called the feasible SIR region. We denote the feasible SIR region or more generally the utility region by $\mathbf{U}$.

It is known that in general the feasible SIR region is not convex, e.g. SIR region in the case of linear receivers, certain rate regions for cellular systems without full coordination, etc. [3]-[5]. Standard properties of the utility sets, like convexity and compactness cannot be taken for granted. However, the region can be "convexified" if the SIRs are expressed in the logarithmic scale, thereby giving rise to the notion of a feasible $\log$-SIR region. The convexity property of the feasible logSIR region is a crucial element in the development of power control strategies.

We investigate the structure of certain utility sets (SIR regions) for the case without power constraints. This not only simplifies the problem but it has the advantage of clearly elucidating the effects of interference coupling on the feasible SIR region and the resulting proportional fair operating point. Analyzing the problem for high signal-tonoise-and-interference-ratio (SINR) often helps better understand its analytical structure [6], providing a basis for future extensions, where noise and power constraints are included. The axiomatic framework is useful for characterizing coupling effects.

As an example, consider the function

$$
\begin{aligned}
& \sum_{k \in \mathcal{K}} \omega_{k} \log \frac{[\boldsymbol{V} \boldsymbol{p}]_{k}}{p_{k}}, p_{k}=\exp \left(s_{k}\right), \boldsymbol{\omega}>\mathbf{0} \\
& \qquad \sum_{k \in \mathcal{K}} \omega_{k}=1,
\end{aligned}
$$

where $\boldsymbol{V}$ is the coupling matrix and $[\boldsymbol{V}]_{k l}$ is the coupling coefficient between the $l^{\text {th }}$ transmitter and $k^{\text {th }}$ receiver. This is the weighted sum of inverse SIRs. We can picture (1) as a loss function. Hence, we would like to minimize this function and we would be interested in exploring the convexity 
properties of such a function. The results in [7] states that (1) is jointly convex with respect to $s$, if and only if $\boldsymbol{V} \boldsymbol{V}^{T}$ is irreducible. $\boldsymbol{V}$ is the link gain matrix for a wireless system. However, the convexity of the function defined in (1) is not sufficient to ensure strict convexity of the log-SIR region. Strict convexity is a very useful property from an optimization perspective. Strict convexity along with certain additional natural properties of interference functions (shall be discussed in detail in Section II-D) allow the existence of a minimal/maximal element. It can be observed, that the joint convexity of (1) does not guarantee that a minimizer to the optimization problem exists, i.e. the infimum need not be equal to the minimum.

We impose an additional property of strict convexity of the interference balancing functions (introduced in Section II-B) to ensure that the log-SIR region is strictly convex. There is no connection (as yet) between the strict convexity of (1) and the strict convexity of the log-SIR region. In [8] only a subset of the family of strict convex sets, specifically a subset of the family of strictly convex log-SIR regions was considered. The considered sets possessed the property, that $\max _{\boldsymbol{u} \in \mathbf{U}} \prod_{k \in \mathcal{K}} u_{k}$ has a strictly positive solution, where $\mathbf{U}$ is the utility region or the feasible SIR region. It is not immediately obvious that these sets are connected with the interference function framework.

In this paper we shall establish this connection between the strict convexity of the log-SIR regions and certain properties of the interference function framework for the case of no power constraints. The contributions and outline of our paper are as follows:

1) We show that under certain conditions for interference functions with the following two properties:

a) strict log-convexity on their respective dependency sets, and

b) and strict positivity on their respective dependency sets (introduced in Section II-D)

we obtain strictly convex sets (strictly convex log-SIR regions). In this case there exists a unique optimizer for the product of utilities maximization problem for these sets, i.e. the infimum is equal to the minimum. We are able to construct the corresponding power vector.

2) In Section III we introduce two graph theoretical representations of interference coupling:

a) the power graph, and

b) the interference graph.

We investigate the relationship between the connectivity of the power graph and the irreducibility of the global dependency matrix $G$ (explained in Section II-C).

3) We investigate the relationship between the connectivity of the interference graph and the irreducibility of the matrix $G G^{T}$. We show that the irreducibility of both $G$ and $G G^{T}$, along with the two properties of the interference function described above are necessary and sufficient conditions, to show the existence of a unique optimizer for the family of strictly convex sets (see Section IV).

We provide a complete characterization of the log-SIR region and extend the results of strict convexity of the feasible log-
SIR region from linear interference functions to a broader class of log-convex interference functions.

The main theorem in this paper has applications to social choice theory and specifically to the existence of a maximal element ${ }^{1}$. Existence of the maximal element follows from the strict convexity of the utility set. The maximal element could be the Nash bargaining solution (NBS) (the NBS is discussed briefly in Section II-A). We provide a constructive proof of the results. We show that we require the reducibility of the global dependency matrix $\boldsymbol{G}$ (explained in detail in Section II-C) to investigate the weak Pareto optimal points ${ }^{2}$. We state the consequences of irreducibility via the combinatorial characterization in Section IV.

\section{INTERFERENCE COUPLED WIRELESS SySTEMS}

Before we begin to describe our system model and present the relevant definitions, we describe certain preliminaries in Section II-A below.

\section{A. Notation and Preliminaries}

Matrices and vectors are denoted by bold capital letters and bold lowercase letters, respectively. Let $\boldsymbol{y}$ be a vector, then $y_{l}=[\boldsymbol{y}]_{l}$ is the $l^{t h}$ component. Likewise let $G_{m n}=[\boldsymbol{G}]_{m n}$ be a component of the matrix $\boldsymbol{G}$. Let $\boldsymbol{y} \geq 0$ imply that $y_{l} \geq 0$ for all components $l$. Let $\boldsymbol{x} \supsetneqq \boldsymbol{y}$ imply component-wise inequality with strict inequality for at least one component. Corresponding definitions hold for the reverse directions. Let $\boldsymbol{x} \neq \boldsymbol{y}$ imply that the vector differs in at least one component. Let $\mathbf{X}$ represent a set. Let $\mathcal{X}$ represent a family of sets. Let the set of non-negative real numbers be denoted as $\mathbb{R}_{+}$. Let the set of positive real numbers be denoted as $\mathbb{R}_{++}$. Let the set of all natural numbers be denoted as $\mathbb{N}$. Let $\underline{e}(r)$ be a $K$-dimensional vector, which is the all-zero vector with the $r^{t h}$ component set to one, i.e. $[\underline{e}(r)]_{k}= \begin{cases}1 & k=r \\ 0 & k \neq r\end{cases}$ A set $\mathbf{U} \subset \mathbb{R}_{++}^{K}$ is said to be (downward)-comprehensive if for all $\boldsymbol{u} \in \mathbf{U}$ and $\hat{\boldsymbol{u}} \in \mathbb{R}_{++}^{K}, \mathbf{0}<\hat{\boldsymbol{u}} \leq \boldsymbol{u}$ implies $\hat{\boldsymbol{u}} \in \mathbf{U}$. In this paper, compact and closed are defined relatively ${ }^{3}$ in $\mathbb{R}_{++}^{K}$. A bargaining solution is a unanimous agreement on certain utilities $\boldsymbol{u}=\left[u_{1}, \ldots, u_{K}\right]$ from a utility set $\mathbf{U}$. The Nash bargaining solution (NBS) corresponds to a Pareto optimal point $\Phi(\mathbf{U})$ characterized by a set of axioms (Nash axioms)[9], [10]. Let the region $\mathbf{U} \subset \mathbb{R}_{++}^{K}$ be compact, convex and comprehensive. Then, the unique NBS fulfilling Nash's axioms is obtained by maximizing the product of the utilities, i.e. $\max _{\boldsymbol{u} \in \mathbf{U}} \prod_{k \in \mathcal{K}} u_{k}$. Since, $\log \max \prod_{k} u_{k}=$ $\max \log \prod_{k} u_{k}=\max \sum_{k} \log u_{k}$, an equivalent optimizer can be found solving $\max _{\boldsymbol{u} \in \mathbf{U}} \sum_{k \in \mathcal{K}} \log u_{k}$. This strategy is related to proportional fairness (PF) [11]. If the set $\mathbf{U}$ is not compact and convex then it is a-priori unclear whether the

\footnotetext{
${ }^{1}$ Let $(\mathbf{V}, \leq)$ be a partially ordered set and $\mathbf{U} \subset \mathbf{V}$. Then, $\boldsymbol{u} \in \mathbf{U}$ is a maximal element of $\mathbf{U}$, if for all $\hat{\boldsymbol{u}} \in \mathbf{U}, \boldsymbol{u} \leq \hat{\boldsymbol{u}}$ implies that $\boldsymbol{u}=\hat{\boldsymbol{u}}$.

${ }^{2}$ For every set compact, comprehensive set $\mathbf{U} \subset \mathbb{R}_{++}^{K}$, the set of weak Pareto optimal points is defined as follows: $W(\mathbf{U}):=\left\{\boldsymbol{u}^{(1)} \in \mathbf{U}\right.$ : such that there is no $\boldsymbol{u}^{(2)} \in \mathbf{U}$ with $\boldsymbol{u}^{(2)}>\boldsymbol{u}^{(1)}$ \}.

${ }^{3} \mathrm{~A}$ set $\mathbf{U} \subset \mathbb{R}_{++}^{K}$ is said to be relatively closed in $\mathbb{R}_{++}^{K}$ if there exists a closed set $\mathbf{A} \subset \mathbb{R}^{K}$ such that $\mathbf{U}=\mathbf{A} \cap \mathbb{R}_{+}^{K}$. This is the case when all the users are active. It is possible to extend this framework to cases where all users need not be active.
} 
maximum $\max _{\boldsymbol{u} \in \mathbf{U}} \sum_{k \in \mathcal{K}} \log u_{k}$ exists. If it exists then it is unclear whether the optimum is really the NBS.

Definition 1. We say that a set $\mathbf{U} \subset \mathbb{R}_{++}^{K}$ is log-convex, if the image set $\log (\mathbf{U})=\{\boldsymbol{q}=\log (\boldsymbol{u}) \mid \boldsymbol{u} \in \mathbf{U}\}$ is convex.

Definition 2. By $\mathcal{S T}$ we denote the family of all closed comprehensive utility sets $\mathbf{U} \in \mathbb{R}_{++}^{K}$ such that $\log (\mathbf{U})$ is a strictly convex set in $\mathbb{R}^{K}$.

By $\mathcal{S} \mathcal{T}_{c}$ we denote the family of all $\mathbf{U} \in \mathcal{S T}$ that are additionally upper bounded.

For bounded sets from $\mathcal{S T}_{c}$, it was shown in [12] that the unique optimizer fulfilling the Nash axioms is always the optimizer of $\max _{\boldsymbol{u} \in \mathbf{U}} \prod_{k \in \mathcal{K}} u_{k}$. In this paper we consider a possibly unbounded set $\mathbf{U} \in \mathcal{S T}$, for which the results in [8] cannot be applied directly. We now present the interference function framework, which is used to capture interference coupling in wireless systems.

\section{B. Interference Functions}

In a wireless system, the users' utilities can strongly depend on the underlying physical layer. The utility under consideration is $\operatorname{SIR}_{k}(\boldsymbol{p})=\frac{p_{k}}{\mathcal{I}_{k}(\boldsymbol{p})}$, for a user $k \in \mathcal{K}$. The function $\mathcal{I}_{k}(\boldsymbol{p})$ yields the interference power experienced by the $k^{\text {th }}$ user. It is function of the transmission powers $\boldsymbol{p}=\left[p_{1}, \ldots, p_{K}\right]^{T}$. Many other performance measures have a direct relationship with SIR. Utility can represent certain arbitrary performance measure, which depends on the SIR by a strictly monotone and continuous function $\phi$ defined on $\mathbb{R}_{+}$. The utility of user $k$ is $u_{k}(\boldsymbol{p})=\phi_{k}\left(\operatorname{SIR}_{k}(\boldsymbol{p})\right)$, where user $k \in \mathcal{K}$. An example of the above case is capacity: $\phi(x)=\log (1+x)$. Note that the effective path gain of user $k$ can be incorporated in the function $\mathcal{I}_{k}(\boldsymbol{p})$ as an additional scaling factor. In this case, $\operatorname{SIR}_{k}(\boldsymbol{p})$ is the ratio of the received power to the interference power. In order to model the interference, we use the axiomatic framework proposed in [1].

Definition 3. We say that $\mathcal{I}: \mathbb{R}_{+}^{K} \mapsto \mathbb{R}_{+}$is an interference function if the following axioms are fulfilled:

$$
\begin{array}{ll}
A 1 & \text { positivity: } \exists \boldsymbol{p}>\mathbf{0} \text { with } \mathcal{I}(\boldsymbol{p})>0 \\
A 2 & \text { scale invariance: } \mathcal{I}(\alpha \boldsymbol{p})=\alpha \mathcal{I}(\boldsymbol{p}) \text { for all } \alpha \geq 0 \\
A 3 & \text { monotonicity: } \mathcal{I}(\boldsymbol{p}) \geq \mathcal{I}(\hat{\boldsymbol{p}}) \text { if } \boldsymbol{p} \geq \hat{\boldsymbol{p}} .
\end{array}
$$

The axiomatic framework $A 1$ to $A 3$ is related with the framework of standard interference functions [13]. The details about the relationship between the model $A 1-A 3$ and Yates' standard interference functions were discussed in [1], [14]. For the purpose of this paper it is sufficient to be aware that there exists a connection between these two models and the results of this paper are applicable to standard interference functions.

We now introduce the function $C(\boldsymbol{\gamma}, \mathcal{I})$, known as the interference balancing function. The function $C(\boldsymbol{\gamma}, \mathcal{I})$ is an indicator of the feasibility of SIR values $\gamma=\left[\gamma_{1}, \ldots, \gamma_{K}\right]^{T}$ for users $k=1, \ldots, K$, respectively.

$$
C(\boldsymbol{\gamma}, \mathcal{I})=\inf _{\boldsymbol{p}>\mathbf{0}} \max _{k \in \mathcal{K}} \frac{\gamma_{k} \mathcal{I}_{k}(\boldsymbol{p})}{p_{k}}
$$

We have the following expression $C(\boldsymbol{\gamma}, \mathcal{I}) \leq 1$, if and only if for any $\epsilon>0$ there exists a power vector $\boldsymbol{p}_{\epsilon}>\mathbf{0}$ such that $\gamma_{k}\left(\boldsymbol{p}_{\epsilon}\right) \geq \gamma_{k}-\epsilon$, for all $k \in \mathcal{K}$. The feasible SIR region is the sub-level set

$$
\mathbf{S}=\left\{\boldsymbol{\gamma} \in \mathbb{R}_{++}^{K} \mid C(\boldsymbol{\gamma}, \mathcal{I}) \leq 1\right\} .
$$

Boundary points of $\mathbf{S}$ are characterized by $C(\boldsymbol{\gamma}, \mathcal{I})=1$.

\section{Interference Coupling}

The structure of the SIR region depends on the interference coupling. For general interference functions it is not obvious as to what would be an appropriate system to define interference coupling. We define the system as "coupled", if there is some arbitrary power vector $\boldsymbol{p}$ such that $\left[\boldsymbol{D}_{\mathcal{I}}(\boldsymbol{p})\right]_{k r}=1$, where for a given $k, r$ we have

$$
\left[\boldsymbol{D}_{\mathcal{I}}(\boldsymbol{p})\right]_{k r}= \begin{cases}1 \quad & \text { if there exists a } \delta_{r}(\boldsymbol{p})>0 \\ & \text { such that the function } \\ & f_{r}(\delta, \boldsymbol{p})=\mathcal{I}_{k}(\underline{\boldsymbol{p}}-\delta \underline{\boldsymbol{e}}(r)) \\ & \text { is strictly monotone decreasing } \\ & \text { for } 0 \leq \delta \leq \delta_{r}(\boldsymbol{p}) \\ 0 \quad & \text { otherwise. }\end{cases}
$$

The matrix $\boldsymbol{D}$ is called the dependency matrix. Thereby, we obtain the global dependency matrix, which is independent of the choice of the power vector $\boldsymbol{p}$ as follows.

Definition 4. Global dependency matrix: $\boldsymbol{G}_{\mathcal{I}}$ is the global dependency matrix, given by

$$
\left[\boldsymbol{G}_{\mathcal{I}}\right]_{k r}= \begin{cases}1 & \text { if there exists a } \boldsymbol{p} \geq \mathbf{0} \text { such that } \\ & {\left[\boldsymbol{D}_{\mathcal{I}}(\boldsymbol{p})\right]_{k r}>0} \\ 0 & \text { otherwise. }\end{cases}
$$

The non-zero entries in $G:=G_{\mathcal{I}}$ mark the transmitter/receiver pairs, which are coupled by interference. A zero entry implies that no interference is received, irrespective of the magnitude of the transmission power.

Example 1. Consider that users are assigned to different orthogonal resources separated by adaptive interference rejection techniques. This coupling model includes the widely used concept of a "link gain matrix" as a special case.

We assume $G$ to be an irreducible matrix [15], pp. 360-361. This implies that each user is interfered by at least one other user. Irreducibility of $G$ is equivalent to strong connectivity of the graph $\mathcal{G}(\boldsymbol{G})$, where $\mathcal{G}(\boldsymbol{G})$ is defined to be the directed graph of $\mathcal{K}$ nodes, in which there is a directed edge leading from node $r \in \mathcal{K}$ to $k \in \mathcal{K}$ if and only if $[\boldsymbol{G}]_{k r}>0$. The matrix $\boldsymbol{G}$ is called the adjacency matrix of the graph $\mathcal{G}(\boldsymbol{G})$. This graph is said to be strongly connected if for each pair of nodes $(k, r)$, there is an uninterrupted sequence of directed edges leading from $r$ to $k$.

Remark 1. Note that the direction matters in the definition of strong connectivity for directed graphs.

We now introduce the dependency set.

Definition 5. Dependency set: $L_{k}$ is the dependency set for user $k$, if $L_{k}=\left\{r \in \mathcal{K} \mid\left[\boldsymbol{G}_{\mathcal{I}}\right]_{k r}=1\right\}$, where $\boldsymbol{G}$ is given by (4). 
This is the set of transmitters, which have an impact on user $k$. We now present an example below, which display the following remark.

Remark 2. Let $\mathcal{I}_{1}, \ldots, \mathcal{I}_{K}$ be linear interference functions, e.g. $\mathcal{I}_{k}(\boldsymbol{p})=\sum_{j \in \mathcal{K}} v_{k j} p_{j}$. Then, this assumption is not always sufficient to ensure that the infimum in (1) is achieved.

Example 2. Consider the case of linear interference functions $\mathcal{I}_{k}(\boldsymbol{p})$. Hence we have that the link gain matrix $\boldsymbol{V}=\boldsymbol{G}$, where $G$ is the global dependency matrix. Consider the case of a system with 4 users. Let

$$
\boldsymbol{V}=\left(\begin{array}{llll}
0 & 1 & 0 & 1 \\
1 & 0 & 0 & 0 \\
1 & 1 & 0 & 1 \\
1 & 1 & 1 & 0
\end{array}\right)
$$

Let $p_{1}, p_{2}, p_{3}, p_{4}>0$ be arbitrary powers of user $1,2,3$ and 4 respectively. Then we have that $\sum_{k=1}^{4} \log \frac{[\boldsymbol{V} \boldsymbol{p}]_{k}}{p_{k}}=\log \frac{p_{2}}{p_{1}}+\log \frac{p_{1}}{p_{2}}+\log \frac{p_{1}+p_{2}+p_{4}}{p_{3}}+$ $\log \frac{p_{1}+p_{2}+p_{3}}{p_{4}}=\log \frac{\left(p_{1}+p_{2}+p_{4}\right)\left(p_{1}+p_{2}+p_{3}\right)}{p_{3} p_{4}}>\log \frac{p_{3} p_{4}}{p_{3} p_{4}}=0$. Let us choose the powers of user 3 and user 4 as $p_{3}, p_{4}>0$ and the powers of user 1 and user 2 as $p_{1}^{(n)}=p_{2}^{(n)}=1 / n$ for $n \in \mathbb{N}$. With the choice of power vector from the previous sentence, we have that $\inf _{\boldsymbol{p}>\mathbf{0}} \sum_{k=1}^{4} \log \frac{[\boldsymbol{V} \boldsymbol{p}]_{k}}{p_{k}} \leq$ $\log \frac{\left(\frac{2}{n}+p_{4}\right)\left(\frac{2}{n}+p_{3}\right)}{p_{3} p_{4}}$, for all choices of $n \in \mathbb{N}$. We now have that $0 \leq \inf _{\boldsymbol{p}>\mathbf{0}} \sum_{k=1}^{4} \log \frac{[\boldsymbol{V} \boldsymbol{p}]_{k}}{p_{k}} \leq \inf _{n \in \mathbb{N}} \log \frac{\left(\frac{2}{n}+p_{4}\right)\left(\frac{2}{n}+p_{3}\right)}{p_{3} p_{4}}=0$. We can observe that $\inf _{\boldsymbol{p}>\mathbf{0}} \sum_{k=1}^{K} \log \frac{[\boldsymbol{V} \boldsymbol{p}]_{k}}{p_{k}}=0$. Let us now assume that there exists a power vector $\tilde{\boldsymbol{p}}>$ $\mathbf{0}$ such that $0=\sum_{k=1}^{4} \log \frac{[\boldsymbol{V} \hat{\boldsymbol{p}}]_{k}}{\hat{p}_{k}}$. Then we have that $0=\log \frac{\left(\hat{p}_{1}+\hat{p}_{2}+\hat{p}_{4}\right)\left(\hat{p}_{1}+\hat{p}_{2}+\hat{p}_{3}\right)}{\hat{p}_{3} \hat{p}_{4}}=\log \left(1+\frac{\left(\hat{p}_{1}+\hat{p}_{2}\right)\left(\hat{p}_{3}+\hat{p}_{4}\right)}{\hat{p}_{3} \hat{p}_{4}}+\right.$ $\left.\frac{\hat{p}_{1}+\hat{p}_{2}}{\hat{p}_{3} \hat{p}_{4}}\right)>\log 1=0$. Hence, we conclude that the infimum is not achieved.

Hence, we impose certain additional properties of interference functions, so that we can achieve the desired infimum. These properties are introduce in Section II-D below.

\section{Additional Properties of Interference Functions}

We begin by introducing the property of strict monotonicity.

Definition 6. Strict monotonicity: An interference function is $\mathcal{I}_{k}(\boldsymbol{p})$ is said to be strictly monotonic on its dependency set $L_{k}$, if $\boldsymbol{p}^{(1)} \geq \boldsymbol{p}^{(2)}$ with $p_{r}^{(1)}>p_{r}^{(2)}$ for some $r \in L_{k}$, then $\mathcal{I}_{k}\left(\boldsymbol{p}^{(1)}\right)>\overline{\mathcal{I}}_{k}\left(\boldsymbol{p}^{(2)}\right)$.

Example 3. Consider the interference function $\mathcal{I}_{k}(\boldsymbol{p})=$ $\sum_{r \in L_{k}} v_{k r} p_{r}$, where $v_{k r} \in \mathbb{R}_{+}$is the link gain between transmitter $r$ and receiver $k$. All users $r \in L_{k}$, where $L_{k}$ is the dependency set interfere with user $k$. Strict monotonicity of $\mathcal{I}_{k}$ implies, that there exists at least one user $r \in L_{k}$ such that $\mathcal{I}_{k}(\boldsymbol{p})$ is strictly increasing with respect to $p_{r}$.

Definition 7. Strict positivity: An interference function $\mathcal{I}_{k}(\boldsymbol{p})$ for $k \in \mathcal{K}$ is said to be strictly positive on its dependency set $L_{k}$, if for $\boldsymbol{p} \geq \mathbf{0}, \boldsymbol{p} \neq[0, \ldots, 0]^{T}$ and $p_{l}>0$ for some $l \in L_{k}$, we have that $\mathcal{I}_{k}(\boldsymbol{p})>0$.

From a practical point of view strict positivity seems very natural in wireless systems. None the less, it is an important mathematical restriction whose impact shall be observed in Section IV.

Remark 3. Strict positivity of the interference function is a stronger condition than A1. Strict positivity of the interference function $\mathcal{I}_{k}(\boldsymbol{p})$ is defined with respect to its respective dependency set $L_{k}$.

Example 4. Consider the following examples: $\mathcal{I}(\boldsymbol{p})=$ $\sum_{k=1}^{K} v_{k} p_{k}, \boldsymbol{v}>\mathbf{0}, \boldsymbol{v} \neq[0, \ldots, 0]^{T}$ is a strict positive interference function; $\mathcal{I}(\boldsymbol{p})=\prod_{k} p_{k}^{\omega_{k}}, \boldsymbol{\omega} \geq \mathbf{0}, \sum_{k=1}^{K} \omega_{k}=1$ is not a strict positive interference function.

Definition 8. Log-convex interference function: An interference function $\mathcal{I}: \mathbb{R}_{+}^{K} \mapsto \mathbb{R}_{+}$is said to be a log-convex interference function if $A 1-A 3$ are fulfilled and $\mathcal{I}(\exp \{s\})$ is log-convex on $\mathbb{R}^{K}$.

Let $f(s):=\mathcal{I}(\exp \{s\})$. The function $f: \mathbb{R}^{K} \mapsto \mathbb{R}_{+}$is $\log$-convex on $\mathbb{R}^{K}$ if and only if $\log f$ is convex or equivalently $f(s(\lambda)) \leq f\left(s^{(1)}\right)^{1-\lambda} f\left(s^{(2)}\right)^{1-\lambda}$, for all $\lambda \in(0,1)$, $\boldsymbol{s}^{(1)}, \boldsymbol{s}^{(2)} \in \mathbb{R}^{K}$, where $\boldsymbol{s}(\lambda)=(1-\lambda) \boldsymbol{s}^{(1)}+\lambda \boldsymbol{s}^{(2)}, \lambda \in(0,1)$. Note that the change of variable $\boldsymbol{p}=\exp \{\boldsymbol{s}\}$ was already used in [16], [17].

Definition 9. Strict log-convexity: A log-convex interference function $\mathcal{I}_{k}$ is said to be strictly log-convex on its dependency set if for all $\boldsymbol{p}^{(1)}, \boldsymbol{p}^{(1)} \in \mathbb{R}_{++}^{K}$, with $p_{l}^{(1)} \neq p_{l}^{(2)}$ for at least one $l \in L_{k}$, we have $\mathcal{I}_{k}(\boldsymbol{p}(\lambda))<\left(\mathcal{I}_{k}\left(\boldsymbol{p}^{(1)}\right)\right)^{1-\lambda}\left(\mathcal{I}_{k}\left(\boldsymbol{p}^{(2)}\right)\right)^{1-\lambda}$, where $\boldsymbol{p}(\lambda)=\boldsymbol{p}^{(1)^{1-\lambda}} \boldsymbol{p}^{(2)^{\lambda}}$.

From Remark 2 we have that linear interference functions always satisfy definitions $6,7,8$. However, they need not satisfy definition 9. The property of strict log-convexity of interference functions on their respective dependency sets will be used to show that the infimum can be achieved. We introduce a table of notation, for quickly reviewing all the properties of interference functions introduced in the paper.

\begin{tabular}{|c|c|}
\hline$\overline{A A 1}$ (Positivity) & $\begin{array}{l}\text { There exists a } \boldsymbol{p}>\mathbf{0} \\
\text { with } \mathcal{I}(\boldsymbol{p})>0\end{array}$ \\
\hline$A 2$ (Scale Invariance) & $\begin{array}{l}\mathcal{I}(\alpha \boldsymbol{p})=\alpha \mathcal{I}(\boldsymbol{p}) \\
\text { for all } \alpha \geq 0\end{array}$ \\
\hline$A 3$ (Monotonicity) & $\mathcal{I}(\boldsymbol{p}) \geq \mathcal{I}(\hat{\boldsymbol{p}})$ if $\boldsymbol{p} \geq \hat{\boldsymbol{p}}$ \\
\hline Strict Monotonicity & $\begin{array}{l}\boldsymbol{p}^{(1)} \geq \boldsymbol{p}^{(2)}, p_{r}^{(1)}>p_{r}^{(2)} \\
\text { for some } r \in L_{k} \\
\text { then } \mathcal{I}_{k}\left(\boldsymbol{p}^{(1)}\right)>\mathcal{I}_{k}\left(\boldsymbol{p}^{(2)}\right)\end{array}$ \\
\hline Strict Positivity & $\begin{array}{l}\boldsymbol{p} \geq \mathbf{0}, \boldsymbol{p} \neq[0, \ldots, 0]^{T} \\
\text { and } p_{l}>0 \text { for } l \in L_{k} \\
\text { then } \mathcal{I}_{k}(\boldsymbol{p})>0\end{array}$ \\
\hline Log-convexity & $\begin{array}{l}f(\boldsymbol{s}):=\mathcal{I}(\exp \{\boldsymbol{s}\}) \\
\text { and } \log f \text { is convex }\end{array}$ \\
\hline Strict Log-convexity & $\begin{array}{l}\text { for all } \boldsymbol{p}^{(1)}, \boldsymbol{p}^{(1)} \in \mathbb{R}_{++}^{K}, \\
\text { with } p_{l}^{(1)} \neq p_{l}^{(2)} \text { for at least } \\
\text { one } l \in L_{k}, \text { we have } \\
\mathcal{I}_{k}(\boldsymbol{p}(\lambda))<\left(\mathcal{I}_{k}\left(\boldsymbol{p}^{(1)}\right)\right)^{1-\lambda} \\
\left(\mathcal{I}_{k}\left(\boldsymbol{p}^{(2)}\right)\right)^{1-\lambda} \text { where } \\
\boldsymbol{p}(\lambda)=\left(\boldsymbol{p}^{(1)}\right)^{1-\lambda}\left(\boldsymbol{p}^{(2)}\right)^{\lambda}\end{array}$ \\
\hline
\end{tabular}




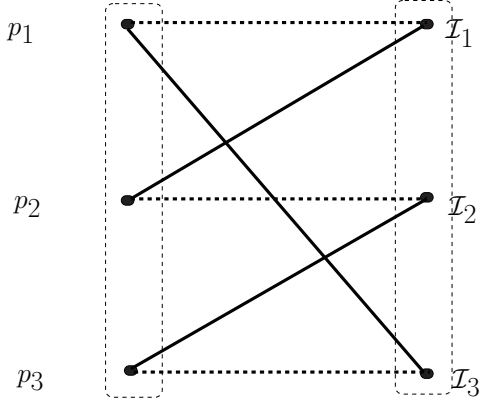

Figure 1. Bipartite graph; shaded edges correspond to connections between power node $p_{k}$ and interference node $\mathcal{I}_{k}$ of user $k$; Power graph for 3 users displays full connectivity, corresponding to irreducible matrix $\boldsymbol{G}$.

\section{COMBINATORIAL CHARACTERIZATION OF INTERFERENCE COUPLING}

In this section we present a combinatorial characterization of interference coupling and its relation to the irreducibility of the matrices $G$ and $G G^{T}$ respectively. For this purpose we shall construct a new graphical representation. We know that the global dependency matrix $G$ is irreducible, if and only if the "classical graph" is connected. Before introducing the power and interference bipartite graphs, we give certain intuition as to what certain concepts in these definitions could look like.

Let $\mathbf{P}$ and $\mathbf{I}$ represent the set power nodes and the set of interference nodes, respectively. In the definition below, a power node $p_{k} \in \mathbf{P}$ could be thought of as an abstraction of the transmit power of user $k$. Similarly, an interference node $\mathcal{I}_{k} \in \mathbf{I}$ could be thought of as an abstraction of the interference experienced by user $k$. Power nodes are not connected to each other. Interference nodes are not connected to each other. If node $p_{k}$ has an undirected edge to certain other interference nodes, e.g. $\mathcal{I}_{k_{1}}, \mathcal{I}_{k_{2}}$, where $k, k_{1}, k_{2} \in \mathcal{K}$, then it implies that user $k$ has the ability to cause interference to users $k_{1}$ and $k_{2}$. Having provided this basic intuition we are now in a position to introduce the power and interference bipartite graphs.

Definition 10. For any given matrix $\boldsymbol{G} \in \mathbb{R}_{+}^{K \times K}$, let $\tilde{\mathcal{G}}(\boldsymbol{G})$ be the (undirected) bipartite graph of $2 K$ nodes divided into two disjoint sets $\mathbf{P}$ and $\mathbf{I}$ (each of cardinality $K$ ) such that, there is no edge between nodes within each of these groups and there is an undirected edge between $p_{l} \in \mathbf{P}$ and $\mathcal{I}_{k} \in \mathbf{I}$ if and only if $[\boldsymbol{G}]_{k l}>0$.

It is worth pointing out that if the power nodes are labeled $\mathbf{P}=\left\{p_{1}, \ldots, p_{K}\right\}$ and the interference nodes are labeled $\mathbf{I}=$ $\left(\mathcal{I}_{1}, \ldots, \mathcal{I}_{K}\right)$ then the adjacency matrix of $\tilde{\mathcal{G}}(\boldsymbol{G})$ is a $2 K \times 2 K$ partitioned matrix of the form $\left(\begin{array}{cc}\mathbf{0} & \boldsymbol{G}^{T} \\ \boldsymbol{G} & \mathbf{0}\end{array}\right)$ [18], page 15 .

Definition 11. Connectivity of $\tilde{\mathcal{G}}(\boldsymbol{G})$ : For any $\boldsymbol{G}$, two nodes $k_{1}$ and $k_{2}$ in $\tilde{\mathcal{G}}(\boldsymbol{G})$ are said to be connected, if and only if there exists a sequence of edges $(i, j) \in \mathbf{P} \times \mathbf{I}$ or $(i, j) \in \mathbf{P} \times \mathbf{I}$ such that $\left\{\left(l_{0}, l_{1}\right),\left(l_{1}, l_{2}\right), \ldots,\left(l_{N-2}, l_{N-1}\right),\left(l_{N-1}, l_{N}\right)\right\}$ with $l_{0}=k_{0}$ and $l_{N}=k_{2}$.

Stated informally, the definition says that $\tilde{\mathcal{G}}$ is connected, if and only if every two distinct nodes are linked by a sequence

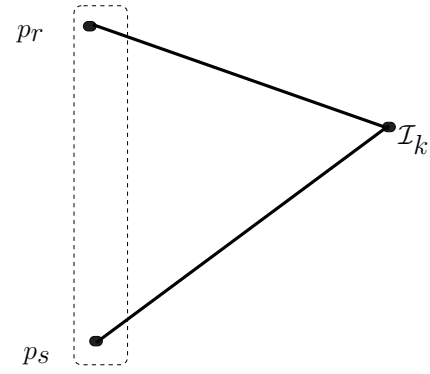

Figure 2. Elementary power step in a Power graph; $p_{r}$ and $p_{s}$ are in $L_{k}^{\prime}$.

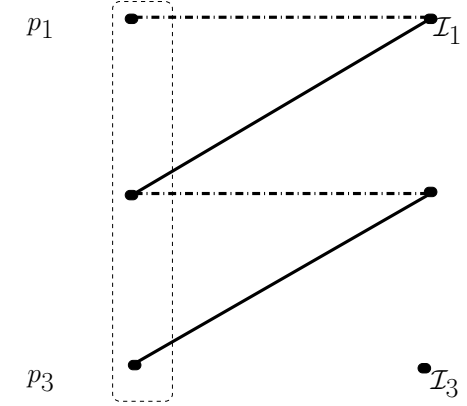

Figure 3. Example of a power graph, which is not fully connected; $L_{k}^{\prime}=$ $\{k\} \cup L_{k}$.

of undirected edges, each of which now connects a power nodes with an interference node. Consider the following matrix

$$
\boldsymbol{G}=\left(\begin{array}{lll}
0 & 1 & 0 \\
0 & 0 & 1 \\
1 & 0 & 0
\end{array}\right)
$$

The bipartite graph for matrix $G$ is displayed in Fig. 1. The dotted edges in Fig. 1 corresponds to an artificial connection in this graph. It does not immediately imply that we have self interference. The presence of self interference shall be discussed in detail in Example 3). We now introduce the power graph and the interference graph. These graphs will help abstract away certain physical layer complications, while capturing interference coupling effects in wireless systems.

\section{A. Power Graph}

We begin by explaining, what we imply by elementary power step. Here we introduce the dependency set $L_{k}^{\prime}$, where $L_{k}^{\prime}=\{k\} \cup L_{k}$.

Definition 12. Elementary power step: An elementary power step is a connection between any two power nodes in the bipartite graph through an interference node.

We can construct an elementary power step between two power nodes $p_{t}$ and $p_{s}$ if and only if $t, s \in L_{k}^{\prime}$, where $L_{k}^{\prime}=$ $\{k\} \cup L_{k}$ (see Fig. 2).

Definition 13. Power graph: We call a bipartite graph of $2 K$ nodes divided into two disjoint sets $\mathbf{P}$ and $\mathbf{I}$ (each of cardinality $K$ ) a power graph if it consists only of elementary 


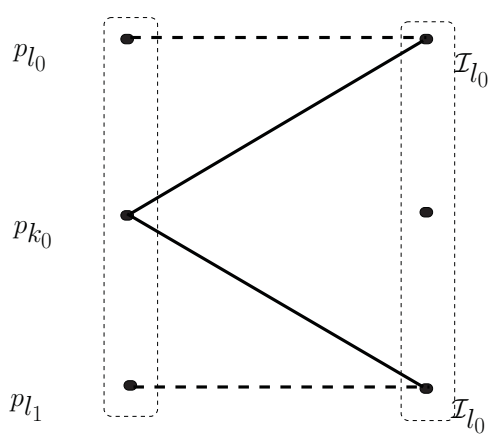

Figure 4. Power of user $k_{0}, p_{k_{0}}$ can be decreased and in turn power of all users in $L_{k_{0}}$ can be decreased.

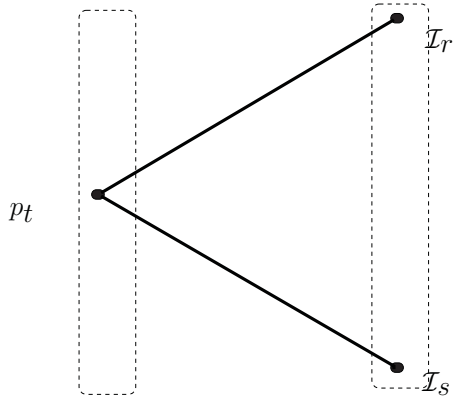

Figure 5. Interference graph; $t \in L_{r}$ and $t \in L_{s}$; Elementary interference step between interference nodes $\mathcal{I}_{r}$ and $\mathcal{I}_{s}$.

power steps. A power path is a path in the power graph made up one or more elementary power steps.

If the power graph is fully connected then each power node can be reached by every other power node. An example of a fully connected power graph is displayed in Fig. 1. An example of a power graph, which is not fully connected is displayed in Fig. 3. We now mention a remark, which will be utilized while proving one of the main results of the paper.

Remark 4 . Let for any user $k_{0}$ the following inequality $p_{k_{0}}>$ $\gamma_{k_{0}} \mathcal{I}_{k_{0}}(\boldsymbol{p})$ hold. Then, we can reduce the power of user $k_{0}$ and in turn reduce the power $p_{l}$ of all users with $k_{0} \in L_{l}$. Let $L_{k_{0}}^{(1)}=\left\{k \mid k_{0} \in L_{k}\right\}$. In the next step we can reduce all the powers $p_{l}$ when there exists a user $k \in L_{k_{0}}^{(1)}$ such that $k \in L_{l}$. Similarly we can construct the dependency set $L_{k_{0}}^{(2)}=\{k \mid$ $\left.\exists k_{1} \in L_{k_{0}}^{(1)}, k_{1} \in L_{k}\right\}$. Using induction we can construct the dependency set $L_{k_{0}}^{(v+1)}=\left\{k \mid \exists k_{1} \in L_{k_{0}}^{(v)}, k_{1} \in L_{k}\right\}$. The power graph is connected, if and only if for each $k_{0} \in \mathcal{K}$ we can find a $v_{0}$ such that $L_{k_{0}}^{\left(v_{0}\right)}=k$.

This is a formal outline of how a power control procedure is regularly carried out in wireless systems. As can be observed, the combinatorial characterization helps to get a better insight into the fashion in which users are coupled by interference, preparing us for interference management and coordination. The irreducibility of $G$ implies the Pareto optimality of the boundary points.

\section{B. Interference Graph}

In this section we discuss the interference graph and the corresponding irreducibility of the matrix $G G^{T}$. We begin by defining an elementary interference step.

Definition 14. Elementary interference step: An elementary interference step is a connection between any two interference nodes in the bipartite graph through a power node.

An elementary interference step between interference nodes $\mathcal{I}_{r}$ and $\mathcal{I}_{s}$ can be constructed if and only if there exists a $t$, $t \in \mathcal{K}$ such that $t \in L_{r} \cap L_{s}$ (see Fig. 5).

Definition 15. Interference graph: We call a bipartite graph of $2 K$ nodes divided into two disjoint sets $P_{r}$ and $I_{c}$ (each of cardinality $K$ ) a interference graph if it consists of only elementary interference steps. An interference path is a path in the interference graph made up of one or more elementary interference steps.

Based on the above definitions we shall prove certain results, which display the relation between the connectivity of the power graph and the irreducibility of the matrix $G$; and the relation between the connectivity of the interference graph and the irreducibility of the matrix $\boldsymbol{G} G^{T}$ in Section IV below. In particular the importance of the interference graph and its interplay with interference functions and the matrix $G G^{T}$ will be visible in the second part of the proof of Theorem 1 .

\section{COMBinatorial Characterization BASED ON INTERFERENCE FUNCTIONS}

We begin by presenting a lemma, which displays the positivity of function $C(\gamma, \mathcal{I})>0$, which is used frequently in our analysis. Here $\gamma:=\gamma(\boldsymbol{u})=\left[\gamma_{1}\left(u_{1}\right), \ldots, \gamma_{K}\left(u_{K}\right)\right]^{T}$ is the SIR vector associated with a utility vector $\boldsymbol{u} \in \mathbf{U}$.

Lemma 1. Let $\mathcal{I}_{1}, \ldots, \mathcal{I}_{K}$ be strictly positive interference functions on their dependency sets $L_{1}, \ldots, L_{K}$, respectively. Then, for all $\gamma_{1}, \ldots, \gamma_{K}>0$ we have that

$$
C(\boldsymbol{\gamma}, \mathcal{I})=\inf _{\boldsymbol{p}>\mathbf{0}} \max _{k \in \mathcal{K}} \frac{\gamma_{k} \mathcal{I}_{k}(\boldsymbol{p})}{p_{k}}>0 .
$$

Proof: We achieve this proof by contradiction. Let $\gamma_{k}>$ 0 , for all $k \in \mathcal{K}$ be such that $C(\gamma)=0$. Then for all $\epsilon>0$ there exists a vector $\boldsymbol{p}(\epsilon), \boldsymbol{p}(\epsilon)>\mathbf{0},\|\boldsymbol{p}\|_{\infty}=1$ (chosen for compactness; it does not alter the problem, since, we are in the case without power constraints) and $\left(\gamma_{k} \mathcal{I}_{k}(\boldsymbol{p}(\epsilon))\right) / p_{k}(\epsilon)<\epsilon$, for $k \in \mathcal{K}$, i.e. $\gamma_{k} \mathcal{I}_{k}(\boldsymbol{p}(\epsilon))<\epsilon p_{k}(\epsilon)$. Let $l_{0}$ be an index with $p_{l_{0}}$ with $p_{l_{0}}(\epsilon)=1$. Let $k_{0}$ be an index such that $\mathcal{I}_{k_{0}}$ is an interference function with $l_{0} \in L_{k_{0}}$. Then we have that $0<c_{3}:=\gamma_{k_{0}} \mathcal{I}_{k_{0}}\left(\boldsymbol{e}\left(l_{0}\right)\right) \leq \gamma_{k_{0}} \mathcal{I}_{k_{0}}(\boldsymbol{p}(\epsilon))<\epsilon p_{k_{0}}(\epsilon)=\epsilon$. We have chosen $\epsilon>0$. Therefore, we have that $0<c_{3} \leq \epsilon$ and $c_{3}=0$, which gives us our required contradiction.

Next, we present a lemma, which insures that strictly logconvex interference functions on their respective dependency sets are also strictly monotonic on their respective dependency sets. This result will be required while investigating the relation between the interference function framework and the family of feasible utility sets.

Lemma 2. Let $\mathcal{I}_{1}, \ldots, \mathcal{I}_{K}$ be strictly log-convex interference functions on their dependency sets $L_{1}, \ldots, L_{K}$, respectively. 
Then, $\mathcal{I}_{1}, \ldots, \mathcal{I}_{K}$ are strictly monotonic on their dependency sets $L_{1}, \ldots, L_{K}$, respectively.

Proof: Refer to [12, Proof of Lemma 5].

Our main resulted presented below, depicts the connection between the family of feasible utilities sets and the interference function framework. We begin with interference functions with the natural properties of strict log-convexity and strict positivity on their respective dependency sets. Then the corresponding global dependency matrix $\boldsymbol{G}$ and $\boldsymbol{G} \boldsymbol{G}^{T}$ are irreducible (i.e. the users in the systems are fully coupled by interference), if and only if the SIR region $\mathbf{S}$ is in the family of strict convex sets $\mathcal{S} \mathcal{T}$. In this case there exists an unique optimum for the problem $\prod_{k \in \mathcal{K}} u_{k}$.

Theorem 1. Let $\mathbf{S}=\left\{\boldsymbol{\gamma} \in \mathbb{R}_{++}^{K} \mid C(\gamma) \leq 1\right\}$, where $C(\gamma)=\inf _{\boldsymbol{p}>\mathbf{0}} \max _{k \in \mathcal{K}}\left(\gamma_{k} \mathcal{I}_{k}(\boldsymbol{p}) / p_{k}\right)$. Let $\mathcal{I}_{k}$ for $k \in \mathcal{K}$ be strictly log-convex and strictly positive interference functions on their respective dependency sets. Then, $\mathbf{S}$ is in the family of sets $\mathcal{S T}$, if and only if $\boldsymbol{G}$ and $\boldsymbol{G G}^{T}$ are irreducible. For this case there exists a unique optimizer $\hat{\boldsymbol{p}}>\mathbf{0}$ for $P F(\mathcal{I})=\inf _{\boldsymbol{p} \in \mathcal{P}} \sum_{k=1}^{K} \log \frac{\mathcal{I}_{k}(\boldsymbol{p})}{p_{k}}$, with an associated SIR vector $\hat{\gamma}$, where $\mathcal{P}$ is the set of power vectors.

Proof: "£": This direction has been proved in [8, Theorem 8].

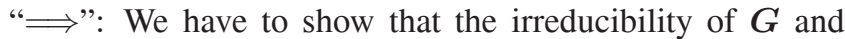
$G G^{T}$ together are necessary and sufficient conditions. We carry out the proof in two steps. Let us assume that $G$ is not irreducible. Then, we show that there are boundary points of $\mathbf{S}$ defined by $\mathbf{S}=\left\{\boldsymbol{\gamma} \in \mathbb{R}_{++}^{K} \mid C(\boldsymbol{\gamma}) \leq 1\right\}$, which are weak Pareto optimal, but not Pareto optimal, i.e. there exists $\gamma^{(1)} \geq$ $\gamma^{(2)}, \gamma^{(1)} \neq \gamma^{(2)}$ with $1=C\left(\gamma^{(1)}\right)=C\left(\gamma^{(2)}\right)$. Since $\boldsymbol{G}$ is reducible, through a permutation of the rows and columns of $\boldsymbol{G}$ we have $\boldsymbol{G}=\left(\begin{array}{cc}\boldsymbol{G}_{(1)} & \mathbf{0} \\ \boldsymbol{G}_{(1,2)} & \boldsymbol{G}_{(2)}\end{array}\right)$, where the matrix $\boldsymbol{G}_{(1)}$ is irreducible. Matrix $\boldsymbol{G}_{(1)}$ is a $k_{1} \times k_{1}$-dimensional matrix. We assume that interference functions $\mathcal{I}_{k}$ for all $k \in \mathcal{K}$ are in the required form. So interference function $\mathcal{I}_{k}$ for all $1 \leq k \leq$ $k_{1}$ is dependent only on $p_{l}$ for $1 \leq l \leq k_{1}$. We now analyze the following equations: $C(\gamma)=\inf _{\boldsymbol{p}>\mathbf{0}} \max _{k \in \mathcal{K}} \gamma_{k} \mathcal{I}_{k}(\boldsymbol{p}) / p_{k}$ and $C\left(\gamma^{(1)}\right)=\inf _{\boldsymbol{p}^{(1)} \in \mathbb{R}^{k_{1}}} \max _{1 \leq k \leq k_{1}} \gamma_{k} \mathcal{I}_{k}(\boldsymbol{p}) / p_{k}$, with $\boldsymbol{p}^{(1)}=\left[p_{1}, \ldots, p_{k_{1}}\right]^{T}$. We also have that $C\left(\gamma^{(2)}\right)=$ $\inf _{\boldsymbol{p}>\mathbf{0}, \boldsymbol{p} \in \mathbb{R}_{+}^{K}} \max _{k_{1}<k \leq K+1} \gamma_{k} \mathcal{I}_{k}(\boldsymbol{p}) / p_{k}$. Then, from Lemma 1 we know that $C\left(\gamma^{(1)}\right)>0, C\left(\gamma^{(2)}\right)>0$ and $C(\gamma)=$ $\max \left(C\left(\gamma^{(1)}\right), C\left(\gamma^{(2)}\right)\right)>0$. We choose $\gamma$ such that $C(\gamma)=C\left(\gamma^{(1)}\right)$. Therefore, we have that $0<C\left(\gamma^{(2)}\right) \leq 1$. For $0<\lambda<1$ we choose $\gamma(\lambda)$ with $\gamma_{k}(\lambda)=\gamma_{k}$, for $1 \leq k \leq k_{1}$ and $\gamma_{k}(\lambda)=\lambda \gamma_{k}$, for $k_{1} \leq k \leq K+1$. Then we have that $C(\gamma(\lambda)) \geq C\left(\gamma^{(1)}(\lambda)\right)=\bar{C}\left(\gamma^{(1)}\right)=$ 1 and $C(\gamma(\lambda))=\inf _{\boldsymbol{p}>\mathbf{0}} \max _{k \in \mathcal{K}}\left(\gamma_{k}(\lambda) \mathcal{I}_{k}(\boldsymbol{p})\right) / p_{k} \leq$ $\inf _{\boldsymbol{p}>\mathbf{0}} \max _{k \in \mathcal{K}}\left(\gamma_{k} \mathcal{I}_{k}(\boldsymbol{p})\right) / p_{k}=1$ and $C(\boldsymbol{\gamma}(\lambda))=1$. Therefore, $\gamma(\lambda)$ is a boundary point. We know that $\gamma(\lambda) \leq \gamma$ and $\gamma(\lambda) \neq \gamma$ for $\lambda \neq 1$. Hence, $\gamma(\lambda)$ is only weak Pareto optimal. We also have for $\gamma(\lambda)=(1-\lambda) \gamma^{(1)}+\lambda \gamma^{(2)}$ that $C(\gamma)=1$, hence $\mathbf{S} \notin \mathcal{S T}$. Therefore, if $\mathbf{S} \in \mathcal{S T}$, then $\boldsymbol{G}$ is irreducible.

Let us assume that matrix $G G^{T}$ is not irreducible, i.e. $G G^{T}$ is reducible. Now we have to show that $\mathbf{S} \notin \mathcal{S T}$. Let $\mathcal{I}_{k}, k \in \mathcal{K}$ be an interference function and $\boldsymbol{p}^{(1)}, \boldsymbol{p}^{(2)}$

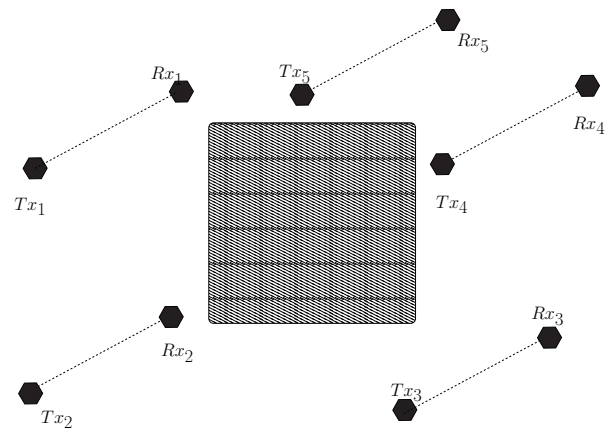

Figure 6. Example of an ad-hoc wireless network with 5 point to point links, where $G$ and $G G^{T}$ are irreducible matrices.

be any two power vectors with $p_{k}^{(1)} \neq p_{k}^{(2)}$. Then we have that $\mathcal{I}_{k}(\boldsymbol{p}(\lambda))<\left(\mathcal{I}_{k}\left(\boldsymbol{p}^{(1)}\right)\right)^{1-\lambda}\left(\mathcal{I}_{k}\left(\boldsymbol{p}^{(2)}\right)\right)^{\lambda}$. Therefore when $\mathcal{I}_{k}(\boldsymbol{p}(\lambda))=\left(\mathcal{I}_{k}\left(\boldsymbol{p}^{(1)}\right)\right)^{1-\lambda}\left(\mathcal{I}_{k}\left(\boldsymbol{p}^{(2)}\right)\right)^{\lambda}$, then we must have that $p_{k}^{(1)}=\mu_{k} p_{k}^{(2)}$. Let $k_{1}$ and $k_{2}$ be any two indices with $L_{k_{1}} \cap L_{k_{2}} \neq \emptyset$. Then $\mu_{k_{1}}=\mu_{k_{2}}$ and $\mu_{k}$ is a constant for all $k \in \mathcal{K}$. When $\boldsymbol{G G}^{T}$ is irreducible, then for all $\boldsymbol{p}^{(1)}, \boldsymbol{p}^{(2)}, \boldsymbol{p}^{(1)} \neq \boldsymbol{p}^{(2)}$ we can find an index $k_{0}$ such that $\mathcal{I}_{k_{0}}\left(\boldsymbol{p}\left(\lambda_{0}\right)\right)<\left(\mathcal{I}_{k}\left(\boldsymbol{p}^{(1)}\right)\right)^{1-\lambda_{0}}\left(\mathcal{I}_{k}\left(\boldsymbol{p}^{(2)}\right)\right)^{\lambda_{0}}$ for some $\lambda_{0}$ (hence for all $\lambda \in(0,1)$ ). This implies that $p_{k_{0}}\left(\lambda_{0}\right) / \mathcal{I}_{k}\left(\lambda_{0}\right)>$ $\left(p_{k_{0}^{(1)}} / \mathcal{I}_{k_{0}}\left(\boldsymbol{p}^{(1)}\right)\right)^{1-\lambda_{0}}\left(p_{k_{0}^{(2)}} / \mathcal{I}_{k_{0}}\left(\boldsymbol{p}^{(2)}\right)\right)^{\lambda_{0}}$. This implies that $\boldsymbol{p}^{(1)}, \boldsymbol{p}^{(2)}$ such that $\boldsymbol{p}^{(1)} \neq \boldsymbol{p}^{(2)}$ such that $\gamma_{k}(\boldsymbol{p}(\lambda))=$ $\left(\gamma_{k}\left(\boldsymbol{p}^{(1)}\right)\right)^{1-\lambda}\left(\gamma_{k}\left(\boldsymbol{p}^{(2)}\right)\right)^{\lambda}$.

Assume that $G$ is irreducible. We can find power vectors $\boldsymbol{p}^{(1)}, \boldsymbol{p}^{(2)}, \boldsymbol{p}^{(1)} \neq \boldsymbol{p}^{(2)}$ such that

$$
\gamma_{k}^{(l)}=\gamma_{k}\left(\boldsymbol{p}^{(l)}\right)=\frac{p_{k}^{l}}{\mathcal{I}_{k}\left(\boldsymbol{p}^{(l)}\right)},
$$

for $l=\{1,2\}$. Let $\gamma^{(1)}$ and $\gamma^{(2)}$ be boundary points. Then $\gamma_{k}(\boldsymbol{p}(\lambda))=\left(\gamma_{k}\left(\boldsymbol{p}^{(1)}\right)\right)^{1-\lambda}\left(\gamma_{k}\left(\boldsymbol{p}^{(2)}\right)\right)^{\lambda}$, for $k \in \mathcal{K}$. Then $\gamma(\lambda)$ is a boundary point. Hence $\mathbf{S} \notin \mathcal{S T}$. So if $\mathbf{S} \in \mathcal{S T}$ then $G G^{T}$ is irreducible.

We now present two corollaries, which display the relationship between the irreducibility of the global dependency matrix $G$ and the matrix $G G^{T}$ with the connectivity of the power graph and the interference graph respectively.

Example 5. Consider an ad-hoc wireless network with 5 point to point links surrounding a building (any object blocking the ability of certain links to interfere with certain other links) as shown in Fig. 6, where $\mathrm{Tx}_{k}$ and $\mathrm{Rx}_{k}$ represent the transmitter and receivers for the $k^{\text {th }}$ link, respectively. The dependency sets of users are as follows: $L_{1}=\{2,5\}, L_{2}=\{1,3\}$, $L_{3}=\{2,4\}, L_{4}=\{3,5\}$ and $L_{5}=\{4,1\}$. We can construct the matrices $\boldsymbol{G}$ and $\boldsymbol{G} \boldsymbol{G}^{T}$ and check, that they are irreducible matrices. If the above case fulfills the conditions of Theorem 1 , then we observe, that even though all the links do not interfere with all the other links, the following statements hold:

- all the boundary points of the resulting SIR set are strictly convex, and

- the proportional fairness resource allocation strategy $P F(\mathcal{I})$ (defined in the statement of Theorem 1) has a unique solution. 
Corollary 1. The global dependency matrix $G$ is irreducible, if and only if we can connect all the power nodes through power paths in the power graph.

Proof: The global dependency matrix $\boldsymbol{G}$ is irreducible if and only if we can find for each pair $(l, k)$ a sequence of natural numbers $k_{0}, k_{1}, \ldots, k_{m}$ such that $k_{0}=l, k_{m}=k$ and $[\boldsymbol{G}]_{k_{r+1} k_{r}}>0$ for all $r=0, \ldots, m-1$. Then we can connect all power nodes $p_{l}$ and $p_{k}$ in the power graph. We illustrate such a connection in the chain below (refer to Remark 4 and first part of proof of Theorem 1 for detailed explanation of the chain).

$$
p_{l} \stackrel{[\boldsymbol{G}]_{k_{1} l}}{\longrightarrow} \mathcal{I}_{k_{1}} \longrightarrow p_{k_{1}} \stackrel{[\boldsymbol{G}]_{k_{2} l}}{\longrightarrow} \mathcal{I}_{k_{2}} \ldots \stackrel{[\boldsymbol{G}]_{k l}}{\longrightarrow} \mathcal{I}_{k} \longrightarrow p_{k}
$$

To prove the opposite direction let us assume that we can connect all the power nodes through the power graph. Then we have that for a pair $(l, k)$ of power nodes we can find a path according to (6). Then we have that $[\boldsymbol{G}]_{k_{0} l}>0$, $[\boldsymbol{G}]_{k_{1} k_{0}}>0, \ldots,[\boldsymbol{G}]_{k_{r+1} k_{r}}>0$ for all $r=0, \ldots, m-1$ and $[\boldsymbol{G}]_{k k_{m-1}}>0$. Hence, we have that the global dependency matrix is irreducible.

Corollary 2. Matrix $G G^{T}$ is irreducible, if and only if we can connect all interference nodes through interference paths in the interference graph.

The next corollary is a direct application of Corollary 1 and Corollary 2 to the case of full self interference. In this special case we display the equivalence between the irreducibility of the global dependency matrix $\boldsymbol{G}$ and the matrix $\boldsymbol{G} \boldsymbol{G}^{T}$.

Corollary 3. Consider a system with full self interference, i.e. $k \in L_{k}$, for all $k \in \mathcal{K}$. For such a system the global dependency matrix $G$ is irreducible, if and only if the matrix $\boldsymbol{G G}^{T}$ is irreducible.

In fact in the case of full self interference, the power graph and the interference graph are equivalent. The dotted edges in the power graph are now solid edges, corresponding to self interference. Under the restriction of full self interference Corollary 3 has shown the equivalence between the irreducibility of the matrices $\boldsymbol{G}$ and $\boldsymbol{G} \boldsymbol{G}^{T}$. This equivalence does not hold in the general case. These insights could better prepare us to design algorithms for interference coordination and management and serve as a building block to study the general case.

We would now like to analyze the case, when the global dependency matrix $G$ is reducible. For a certain SIR vector $\gamma>\mathbf{0}$ with the corresponding interference balancing function $C(\gamma, \mathcal{I})=0$ we consider the following set of power vectors

$$
\mathbf{P}(\gamma):=\left\{\boldsymbol{p} \in \mathbb{R}_{++}^{K} \mid \frac{p_{k}}{\mathcal{I}_{k}(\boldsymbol{p})} \geq \gamma_{k}, \forall k \in \mathcal{K}\right\} .
$$

There is a possibility that the set of powers $\mathbf{P}(\gamma)=\emptyset$. We analyze this case, when the power set $\mathbf{P}(\gamma) \neq \emptyset$. The next result shows that for strictly positive and strictly log-convex interference functions on their respective dependency sets, the irreducibility of the matrices $\boldsymbol{G}$ and $\boldsymbol{G} \boldsymbol{G}^{T}$ are necessary and sufficient conditions for the considered utility sets to be strictly convex. In this case we have shown that there exists a unique optimum for the product of utilities maximization problem.
Theorem 2. Let there exist a SIR vector $\gamma$ such that the power set (defined in (7)) $\mathbf{P}(\gamma) \neq \emptyset$. Then, there exists a set $\mathcal{K}_{1} \subseteq$ $\mathcal{K}=\{1, \ldots, K\}$ and a power vector $\tilde{\boldsymbol{p}} \in \mathbf{P}(\gamma)$ such that $\frac{p_{k}}{\mathcal{I}_{k}(\boldsymbol{p})}=\gamma_{k}$, for all users $k \in \mathcal{K}_{1}$ and for all power vectors $\boldsymbol{p} \in \mathbf{P}(\gamma), \frac{\tilde{p}_{k}}{\mathcal{I}_{k}(\tilde{\boldsymbol{p}})}>\gamma_{k}$, for all users $k \in \mathcal{K}_{2}=\mathcal{K} \backslash \mathcal{K}_{1}$. Then, we have that $\cup_{k \in \mathcal{K}_{1}} L_{k}=\mathcal{K}_{1}$.

Proof: The proof of the prerequisite conditions is discussed in [19], Appendix J, pages 5488-5489. To prove that $\cup_{k \in \mathcal{K}_{1}} L_{k}=\mathcal{K}_{1}$ we begin by showing that $\cup_{k \in \mathcal{K}_{1}} L_{k} \subseteq$ $\mathcal{K}_{1}$. For the sake of obtaining a contradiction assume that $\cup_{k \in \mathcal{K}_{1}} L_{k} \subseteq \mathcal{K}_{1}$ is not true. Then there exists an index $k_{0}$ such that $k_{0} \in \cup_{k \in \mathcal{K}_{1}}$ and $k_{0} \notin \mathcal{K}_{1}$. Let us choose a set $\mathcal{K}_{2}$ such that $k_{0} \in \mathcal{K}_{2}$. We can construct a new power vector such that

$$
\hat{p}_{k_{0}}<\tilde{p}_{k_{0}}, \quad \frac{\hat{p}_{k_{0}}}{\mathcal{I}_{k}(\hat{\boldsymbol{p}})}>\gamma_{k_{0}} .
$$

For an index $k \neq k_{0}$ we define a power vector $\hat{\boldsymbol{p}}$, such that

$$
\hat{p}_{k}=\tilde{p}_{k}, \quad \hat{\boldsymbol{p}} \leq \tilde{\boldsymbol{p}} .
$$

Then from (8), (9) and $A 2$ we have that $\frac{\tilde{p}_{k_{0}}}{\mathcal{I}_{k_{0}}(\hat{\boldsymbol{p}})}>\frac{\hat{p}_{k_{0}}}{\mathcal{I}_{k_{0}}(\hat{\boldsymbol{p}})}>$ $\gamma_{k_{0}}$ and $\frac{\hat{p}_{k}}{\mathcal{I}_{k}(\hat{\boldsymbol{p}})}>\gamma_{k}$. Now we choose an index $k_{1} \in \mathcal{K}_{1}$ and $k_{0} \notin \mathcal{K}_{1}$. Since we have that $\mathcal{I}_{k_{0}}(\tilde{\boldsymbol{p}})>\mathcal{I}_{k_{1}} \hat{\boldsymbol{p}}$, we can reduce the power $\tilde{p}_{k_{1}}$ in the following manner. We choose a power vector $\hat{\boldsymbol{p}}^{(1)}$ such that $\hat{p}_{k_{1}}>\gamma_{k_{1}} \mathcal{I}_{k_{1}}(\tilde{\boldsymbol{p}})$. We choose $\hat{p}_{k}^{(1)}=\tilde{p}_{k}$ for $k \neq k_{1}$ such that $\hat{\boldsymbol{p}}^{(1)} \leq \tilde{\boldsymbol{p}}$ and power vector $\hat{\boldsymbol{p}}^{(1)} \in \mathbf{P}(\gamma)$. Then we have that $\hat{p}_{k_{1}}>\gamma_{k_{1}} \mathcal{I}_{k_{1}}\left(\hat{\boldsymbol{p}}^{(1)}\right)$, for all $k_{1} \in \mathcal{K}_{1}$, which is in contradiction with $\cup_{k \in \mathcal{K}_{1}} L_{k} \subseteq \mathcal{K}_{1}$.

To prove the opposite direction we have to show that $\mathcal{K}_{1} \subseteq$ $\cup_{k \in \mathcal{K}_{1}} L_{k}$. Assume for the sake of obtaining a contradiction that $\mathcal{K}_{1} \subseteq \cup_{k \in \mathcal{K}_{1}} L_{k}$ is not true. Then there exists an index $k_{0} \in \mathcal{K}_{1}$ with $k_{0} \notin \cup_{k \in \mathcal{K}_{1}} L_{k}$. We have that $\tilde{p}_{k_{0}}=\gamma_{k_{0}} \mathcal{I}_{k_{0}}(\tilde{\boldsymbol{p}})$. Since we know that $k_{0} \notin \cup_{k \in \mathcal{K}_{1}} L_{k}$ we have that the power of user $k_{0}, \tilde{p}_{k_{0}}$ has no influence on the interference function $\mathcal{I}_{k}$ of user $k$ with $k \in \mathcal{K}_{1}$. However we are aware that $p_{k_{0}}$ has an impact on certain interference functions $\mathcal{I}_{k}$ with $k \in \mathcal{K}_{2}$. For $k \in \mathcal{K}_{2}$ we have that $\gamma_{k}<\frac{\tilde{p}_{k}}{\mathcal{I}_{k}(\tilde{\boldsymbol{p}})}$. We can now increase the power $\tilde{p}_{k_{0}}$ of user $k_{0}$ such that $\tilde{\boldsymbol{p}} \in \mathbf{P}(\boldsymbol{\gamma}), \hat{p}_{k_{0}}>\tilde{p}_{k_{0}}$, $\hat{p}_{k}^{(2)}=\tilde{p}_{k}$, for $k \in \mathcal{K} \backslash\left\{k_{0}\right\}$ and $\gamma_{k}<\frac{\hat{p}_{k}^{(2)}}{\mathcal{I}_{k}\left(\hat{\boldsymbol{p}}^{(2)}\right)}$, for all $k \in \mathcal{K}_{2}$. Now we have that $\frac{\tilde{p}_{k}^{(2)}}{\mathcal{I}_{k}\left(\hat{\boldsymbol{p}}^{(2)}\right)}>\gamma_{k}, k \in \mathcal{K}_{2} \cup\left\{k_{0}\right\}$, which is in contradiction to $\frac{\mathcal{L}_{k}}{\mathcal{I}_{k}(\boldsymbol{p})}=\gamma_{k}$, for all users $k \in \mathcal{K}_{1}$ and for all power vectors $\boldsymbol{p} \in \mathbf{P}(\gamma)$ and $k_{0} \in \mathcal{K}_{1}$. This implies that $\cup_{k \in \mathcal{K}_{1}} L_{k} \subseteq \mathcal{K}_{1}$. From $\cup_{k \in \mathcal{K}_{1}} L_{k} \subseteq \mathcal{K}_{1}$ and $\mathcal{K}_{1} \subseteq \cup_{k \in \mathcal{K}_{1}} L_{k}$ we have the desired result.

We now return to the point (5) in the proof of Theorem 1. Recollect that, we are in the case when the global dependency matrix $G$ is reducible. In the result below, we show that for all power vectors in the set $\mathbf{P}(\gamma)$, where $\mathbf{P}(\gamma)$ is not empty, the power vectors can be constructed as scaled versions of each other for all users in $\mathcal{K}_{1}$, where $\frac{p_{k}}{\mathcal{I}_{k}(\boldsymbol{p})}=\gamma_{k}$, for all users $k \in \mathcal{K}_{1}$ (for more details on $\mathcal{K}_{1}$ refer to the statement of Theorem 2).

Corollary 4. For all power vectors $\boldsymbol{p}^{(1)}, \boldsymbol{p}^{(2)} \in \mathbf{P}(\gamma)$, there exists a scalar $\mu>0$ with $p_{k}^{(2)}=\mu p_{k}^{(1)}$, for all $k \in \mathcal{K}_{1}$.

Proof: Choose two arbitrary power vectors $\boldsymbol{p}^{(1)}, \boldsymbol{p}^{(1)} \in$ $\mathbf{P}(\gamma)$. For $0<\lambda<1$, we choose $\mathbf{P}(\lambda)$ such that $p_{k}(\lambda)=$ 
$\left(p_{k}^{(1)}\right)^{1-\lambda}\left(p_{k}^{(1)}\right)^{\lambda}$, for all $k \in \mathcal{K}$. Then we have that $\boldsymbol{p}(\lambda) \in$ $\mathbf{P}(\lambda)$ for all $0 \leq \lambda \leq 1$. From Theorem 2 we have for all $k \in \mathcal{K}_{1}$ that $\mathcal{I}_{k}(\boldsymbol{p}(\lambda))=\left(\mathcal{I}_{k}\left(\boldsymbol{p}^{(1)}\right)\right)^{1-\lambda}\left(\mathcal{I}_{k}\left(\boldsymbol{p}^{(2)}\right)\right)^{\lambda}$. Then there exists a scalar $\mu>0$ such that for all $l \in \mathcal{K}_{1}=\cup_{k \in \mathcal{K}_{1}} L_{k}$ we have that $p_{l}^{(2)}=\mu p_{l}^{(1)}$. This shows that for an arbitrary choice of power vectors $\boldsymbol{p}^{(1)}, \boldsymbol{p}^{(2)} \in \mathbf{P}(\boldsymbol{\lambda})$ there exists a scalar $\mu>0$ such that $p_{l}^{(2)}=\mu p_{l}^{(1)}$ for all $l \in \mathcal{K}_{1}$.

We have provided a combinatorial characterization of interference coupling in terms of the interference functions. We know that the interference balancing function $C(\gamma, \mathcal{I})$ is itself an interference function. If interference functions $\mathcal{I}_{k}$ for all $k \in \mathcal{K}$ are log-convex then the corresponding interference balancing function $C(\gamma, \mathcal{I})$ is log-convex. We now briefly discuss the combinatorial characterization of interference coupling in terms of interference balancing functions and its relation to the property of strict log-convexity.

Corollary 5. The interference balancing function $C(\boldsymbol{\gamma}, \mathcal{I})$ is strict log-convex if and only if $\boldsymbol{G}$ and $\boldsymbol{G G}^{T}$ are irreducible matrices, where $G$ is the global dependency matrix.

The result shows that the interference balancing function $C(\gamma, \mathcal{I})$ is strictly monotonic with respect to the SIR vector $\gamma$, if and only if the global dependency matrix $G$ is irreducible. We know that strict log-convexity of an interference function implies strict monotonicity of the interference function. However, we cannot conclude the converse. If we have strict monotone interference balancing function $C(\gamma, \mathcal{I})$ and strict convexity of $\log \left(\mathcal{I}_{k}(\exp (s)) / \exp \left(s_{k}\right)\right)$, then both conditions together are equivalent to the strict convexity of the log-SIR region. This follows from the fact that the first condition is equivalent to the irreducibility of the global dependency matrix $G$ and that the second condition is equivalent to the irreducibility of the matrix $G G^{T}$.

Corollary 6. Interference balancing function $C(\boldsymbol{\gamma}, \mathcal{I})$ is strictly log-convex if and only if the corresponding log-SIR set is strictly convex.

Example 6. For a 2 user system, let $C(\gamma, \mathcal{I})$ be a strictly monotonic interference balancing function, which does not possess the property of being strictly log-convex. Let $\mathcal{I}_{1}(\boldsymbol{p})=$ $p_{2}$ and $\mathcal{I}_{2}(\boldsymbol{p})=p_{1}$. Since we have linear interference functions the corresponding link gain matrix $\boldsymbol{V}=\boldsymbol{G}$ is given by $\boldsymbol{V}=\left(\begin{array}{ll}0 & 1 \\ 1 & 0\end{array}\right)$. Interference balancing function $C(\gamma, \mathcal{I})=\gamma_{1} \gamma_{2}$ and is strictly monotonic. Let SIR of user 1 be $\gamma_{1}=\exp \left(q_{1}\right)$. Then we have that $\log C(\exp (\boldsymbol{q}), \mathcal{I})=$ $q_{1}+q_{2}$.

\section{Conclusions}

We have provided a combinatorial characterization of interference coupling in wireless systems. We have introduced the power graph and interference graph and displayed the relation between their connectivity and the irreducibility of the global dependency matrix $G$ and the matrix $G G^{T}$ respectively. We have proved that for strict positive and strict log-convex interference functions on their respective dependency sets the irreducibility of the matrices $G$ and $G G^{T}$ are necessary and sufficient conditions for the considered utility sets to be strict convex. We have provided a characterization of the log-SIR region, extending results of strict convexity of the feasible logSIR region from linear interference functions to a broader class of log-convex interference functions and have elucidated the importance of the property of log-convexity.

\section{REFERENCES}

[1] M. Schubert and H. Boche, "QoS-based resource allocation and transceiver optimization," Foundations Trends Commun. Inf. Theory, 2005/2006, vol. 2, no. 6, pp. 383-529.

[2] S. Verdu, Multiuser Detection. Cambridge University Press, 1998

[3] H. Boche and S. Stanczak, "Convexity of some feasible QoS regions and asymptotic behavior of the minimum total power in CDMA systems," IEEE Trans. Commun., vol. 52, no. 12, pp. 2190-2197, Dec. 2004.

[4] S. Stanczak and H. Boche, "The infeasible SIR region is not a convex set," IEEE Trans. Commun., vol. 54, no. 11, pp. 1905-1907, Nov. 2006.

[5] R. Cruz and A. Santhanam, "Optimal routing, link scheduling and power control in multi-hop wireless networks," in Proc. 22nd IEEE Conf. Comput. Commmun., Mar. 2003.

[6] L. Zheng and D. Tse, "Diversity and multiplexing: a fundamental tradeoff in multiple-antenna channels," IEEE Trans. Inf. Theory, vol. 49, no. 5, pp. 1073-1096, May 2003.

[7] H. Boche and S. Stanczak, "Strict convexity of the feasible log-SIR region," IEEE Trans. Commun., vol. 56, no. 9, pp. 1-8, Sep. 2008.

[8] H. Boche and M. Schubert, "Nash bargaining and proportional fairness for wireless systems," IEEE/ACM Trans. Netw., vol. 17, no. 5, pp. 14531466, Oct. 2009.

[9] J. F. Nash, "The bargaining problem," Econometrica, vol. 18, pp. 155162, 1950.

[10] H. J. M. Peters, Axiomatic Bargaining Game Theory. Kluwer Academic Publishers, 1992.

[11] F. P. Kelly, A. K. Maulloo, and D. K. H. Tan, "Rate control in communication networks: shadow prices, proportional fairness and stability," J. Operational Research Society, vol. 49, pp. 237-252, 1998.

[12] H. Boche and M. Schubert, "A generalization of Nash bargaining and proportional fairness to log-convex utility sets with power constraints," IEEE Trans. Inf. Theory, 2010, in print.

[13] R. D. Yates, "A framework for uplink power control in cellular radio systems," IEEE J. Sel. Areas Commun., vol. 13, no. 7, pp. 1341-1348, Sep. 1995.

[14] H. Boche and M. Schubert, "A unifying approach to interference modeling for wireless networks," IEEE Trans. Signal Processs., vol. 58, no. 6, June 2010

[15] R. Horn and C. Johnson, Matrix Analysis. Cambridge University Press, 1985.

[16] C. W. Sung, "Log-convexity property of the feasible SIR region in power-controlled cellular systems," IEEE Commun. Lett., vol. 6, no. 6, pp. 248-249, 2002.

[17] D. Catrein, L. A. Imhof, and R. Mathar, "Power control, capacity, and duality of uplink and downlink in cellular CDMA systems," IEEE Trans. Commun., vol. 52, no. 10, pp. 671-687, 2004.

[18] D. Svetkovic, M. Doob, and H. Sachs, Spectra of Graphs. Academic Press, 1978 .

[19] H. Boche and M. Schubert, "A calculus for log-convex interference functions," IEEE Trans. Inf. Theory, vol. 54, no. 12, pp. 5469-5490, Dec. 2008.

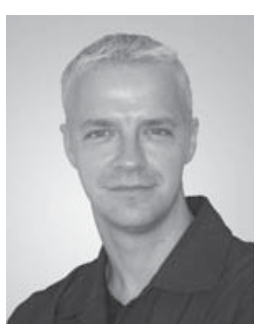

Holger Boche (M'04-SM'07-F'11) received the Dipl.-Ing. and Dr.-Ing. degrees in electrical engineering from the Technische Universitaet Dresden, Dresden, Germany, in 1990 and 1994, respectively. He graduated in mathematics from the Technische Universitaet Dresden in 1992. From 1994 to 1997, he did postgraduate studies in mathematics at the Friedrich-Schiller University Jena, Jena, Germany. He received his Dr. rer. nat. degree in pure mathematics from the Technische Universitaet Berlin, Berlin, Germany, in 1998. In 1997, he joined the Heinrich-Hertz-Institut (HHI) Nachrichtentechnik Berlin, Berlin, Germany. Since 2002, he has been a Full Professor for mobile communication networks with the Institute for Communications Systems, Technische University Berlin. In 2003, he became Director of the Fraunhofer German-Sino Lab for Mobile Communications, Berlin, Germany, and since 2004 he has also been Director of the Fraunhofer Institute for Telecommunications (HHI), Berlin, Germany. Since, October 2010 he is with the Institute of Theoretical Information Technology and Full Professor at the Technical University of Munich, Munich, 
Germany. He was a Visiting Professor with the ETH Zurich, Zurich, Switzerland, during the 2004 and 2006 Winter terms, and with KTH Stockholm, Stockholm, Sweden, during the 2005 Summer term. Prof. Boche is a Member of IEEE Signal Processing Society SPCOM and SPTM Technical Committee. He was elected a Member of the German Academy of Sciences (Leopoldina) in 2008 and of the Berlin Brandenburg Academy of Sciences and Humanities in 2009. He received the Research Award Technische Kommunikation from the Alcatel SEL Foundation in October 2003, the Innovation Award from the Vodafone Foundation in June 2006, and the Gottfried Wilhelm Leibniz Prize from the Deutsche Forschungsgemeinschaft (German Research Foundation) in 2008. He was co-recipient of the 2006 IEEE Signal Processing Society Best Paper Award and recipient of the 2007 IEEE Signal Processing Society Best Paper Award.

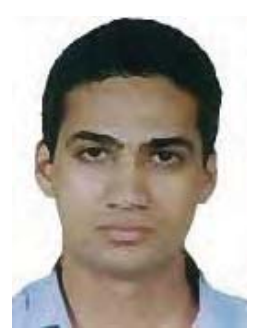

Siddharth Naik received his MS in Electrical Engineering with a specialization in Wireless Systems from the Royal Institute of Technology (KTH), Stockholm, Sweden in 2004. He was with the SPANN Lab, IIT Bombay, India in 2004 and 2005. Since, October 2005 he has been the Fraunhofer German-Sino Lab for Mobile Communications, Berlin, Germany. Since January 2008 he has been a research associate at the Technical University of Berlin, Berlin, Germany. Since October 2010 he has been research associate with the Institute of Theoretical Information Technology at the Technical University of Munich, Munich, Germany. His research interests are game theory, mechanism design, probability theory, stochastic processes, microeconomic theory and optimization.

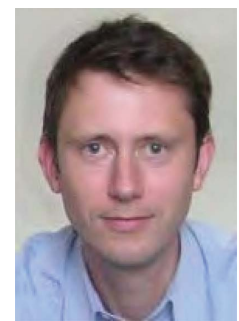

Martin Schubert received the diploma and doctoral degrees in electrical engineering from the Technische Universitaet, Berlin, Germany, in 1998 and 2002, respectively. In 1998 he joined the Heinrich-Hertz Institute for Telecommunications (HHI), Berlin, as a Research Assistant. Since 2003, he has been with the Fraunhofer GermanSino Lab for Mobile Communications (MCI), where he is working as a Senior Researcher. He is also Lecturer at the Technical University of Berlin. He was co-organiser of the VDE/ITG Workshop on Smart Antennas 2009 in Berlin. His current research interests include the modelling and optimisation of interference-coupled wireless networks, and the development of iterative algorithms for adaptive interference mitigation and resource allocation. Dr. Schubert was a corecipient of the VDE JohannPhilipp-Reis Award in 2007. He coauthored the 2007 Best Paper Award of the IEEE Signal Processing Society. Since 2009 he is Associate Editor of the IEEE TRANSACTIONS OF Signal PRocessing. 